\title{
Tumor-targeting, Systemically Delivered Antisense HER-2 Chemosensitizes Human Breast Cancer Xenografts Irrespective of HER-2 Levels
}

\author{
Antonina S. Rait, Kathleen F. Pirollo, Laiman Xiang, David Ulick, and Esther H. Chang \\ Department of Oncology, Lombardi Cancer Center, Georgetown University Medical Center, Washington, \\ DC, USA
}

Accepted July 1, 2002

\begin{abstract}
Background: The failure to respond to chemotherapy is a major obstacle in the successful treatment of breast cancer. We have previously shown that anti-HER-2 antisense oligonucleotide (AS HER-2 ODN) treatment was able to sensitize breast cancer cells to various chemotherapeutic agents in vitro irrespective of their HER-2 status, indicating that the use of AS HER-2 ODN therapy for breast cancer is not limited to tumors overexpressing the protein. One of the main drawbacks to the use of antisense therapy in the clinical setting is the lack of an efficient, tumor-targeting, systemic delivery method. We have developed a tumor-specific, ligand-targeting, cationic liposome delivery system designed for systemic gene therapy of cancer. In this study we employ this ligand-liposome strategy to enhance the delivery of the AS Her-2 ODN to breast cancer cells, including those that do not overexpress HER-2, in vitro and in vivo.

Materials and Methods: A cationic liposome complex that includes folate as the targeting ligand was designed and optimized for more efficient delivery of AS HER-2 ODN to breast tumors cells in vitro, and more significantly, for systemic delivery with tumor-specific targeting in vivo. Human breast cancer cell line MDAMB-435, which does not overexpress HER-2, was used to compare the degree of chemosensitization to the taxanes of AS HER-2 ODN delivered via the optimized folateliposome versus commercial Lipofectin. MDA-MB-435 xenograft tumors were also used to evaluate the anti-tumor
\end{abstract}

effect of the combination of systemically delivered folateliposome-AS HER-2 ODN and docetaxel (Taxotere). Results: The optimized folate-liposome-AS HER-2 ODN complex significantly increases the response of breast tumor cell lines to conventional chemotherapeutic agents in vitro as compared to AS HER-2 delivered via an unliganded commercially available reagent, Lipofectin. In vivo, the folate-liposome-AS HER-2 ODN complex has prolonged stability in blood and increased uptake in tumors. More significantly, the combination of intravenously administered ligand-liposome-AS HER-2 ODN and docetaxel resulted in a marked inhibition of xenograft growth in an aggressive breast cancer model that does not overexpress HER-2, even after treatment ended.

Conclusions: Although there are other reports of liposomal delivery of AS ODNs, this is the first report of in vivo efficacy against human cancer cells using a tumor-targeting liposome delivery system for systemic AS therapy. Moreover, the increased stability in circulation and anti-tumor efficacy observed were obtained without the need for continuous intravenous infusion. HER-2 is an integral component within a network of cell growth pathways that can affect many different types of tumors where HER-2 may be a contributing factor, such as ovarian, esophageal, and GI malignancies including colon and pancreatic cancers. Therefore, the effectiveness of this therapy with xenograft tumors that do not overexpress HER-2 has the potential to expand the clinical usefulness of this efficacious form of therapy.

\section{Introduction}

The HER-2 (c-erbB-2) gene is a member of a family of transmembrane receptor tyrosine kinases that also includes c-erbB-1 (EGF-R), c-erbB-3, and c-erbB-4. Although all four family members and their ligands are associated with cancers of diverse origins (reviewed in 1), HER-2 has been found to be the EGF-R family member most relevant in human cancers. Amplification and overexpression of HER-2 have

A. S. R. and K. F. P. contributed equally to this manuscript. Address correspondence and reprint requests to: E. H. Chang, Georgetown University Medical Center, Lombardi Cancer Center, Research Building, E420, 3970 Reservoir Road NW, Washington, DC 20057-1469. Phone: 202-687-8418; fax: 202-687-8434; e-mail: change@georgetown.edu. been reported in a number of tumor types including ovary, lung, salivary gland, kidney, bladder, and particularly in breast tumors (reviewed in 1, 2), where it has been identified in almost one-third of breast cancer patients. Overexpression of the HER-2 protein has also been associated with more aggressive tumor growth, increased metastatic potential, and poorer patient prognosis $(3,4)$. Because of its strong association with disease, HER-2 has been the target for development of therapeutic agents using various strategies. These include the use of antibodies against HER-2 such as the humanized monoclonal antibody Herceptin. Herceptin has shown some efficacy against chemoresistant metastatic breast cancers in Phase II clinical trials when combined with cisplatin and other chemotherapeutic 
agents (5-7). The selective expression of suicide genes driven by the HER-2 promoter (8) and the use of the adenovirus EIA protein to repress HER-2 expression (9) are also among the strategies being developed.

Sequence-specific antisense HER-2 oligonucleotides (AS HER-2 ODN) that interfere with HER-2 mRNA translation are also potential therapeutic agents. We have previously demonstrated the effectiveness of AS HER-2 ODN in sensitizing breast cancer cells to various chemotherapy agents in vitro (10). This sensitization was found to be independent of the HER-2 status of the cells, possibly reflecting the central role HER-2 plays in signaling through the other members of the c-erb-B receptor family.

The potential of AS technology as a therapeutic agent has been recognized for some time. However, the lack of an efficient in vivo systemic delivery system for these ODNs has limited their clinical use. A nonviral, ligand-targeting, cationic liposomal systemic delivery system has been optimized in our laboratory for targeted gene therapy of cancer. This ligand-liposome complex has been shown to preferentially target tumor cells due to elevated levels of the corresponding receptor on their surface. High levels of expression of the ligand-liposome delivered gene were evident in both primary tumors and metastasis, but not in normal tissue such as liver, lung, bone marrow, and intestinal crypts $(11,12)$. In in vitro and in vivo experiments, we have shown that restoration of wild-type $\mathrm{p} 53$ function by means of this systemically delivered tumor-targeted complex renders the tumor cells more sensitive to conventional chemotherapeutic agents or radiation in head and neck, prostate, breast, and other tumor models (11-14).

In this study we employed our liposome complex, targeted by the addition of the folate ligand, to deliver an AS HER-2 ODN to breast cancer cells in vitro and in vivo. We demonstrated that this folateliposome-AS HER-2 ODN complex improved tumor uptake, and increased ODN stability in blood, when compared to the free ODN. Moreover, intravenous administration of the folate-liposome-AS HER-2 complex sensitized MDA-MB-435 xenograft tumors that have a low level of HER-2 expression, to the effects of the drug docetaxel (Taxotere), resulting in significant inhibition of tumor growth during and even after the end of treatment. Continuous intravenous infusion was not required. Thus, the use of the folate-liposome complex as an efficient, systemic delivery vehicle for antisense molecules shows promise as a means to effectively employ antisense technology as a molecular therapy for both primary and metastatic breast cancer in a wide range of patients.

\section{Methodology}

Cell Lines and Culture

Human breast carcinoma cell line MDA-MB-435 was provided by the GUMC Tissue Culture Core Facility. A folate independent derivative of this cell line (MDA-MB-435 FF) was developed by culturing the cells in folate-free medium (RPMI) for 3 weeks. H500 is a normal human fibroblast cell line established from an infant foreskin. Cell culture media and medium ingredients were obtained from Biofluids (Rockville, MD, USA). MDA-MB-435 was cultured in improved MEM (IMEM) supplemented with $10 \%$ heat-inactivated fetal bovine serum (FBS), $2 \mathrm{mM} \mathrm{L}$-glutamine and $50 \mu \mathrm{g} / \mathrm{ml}$ each of penicillin, streptomycin, and neomycin. MDA-MB$435 \mathrm{FF}$ cells were cultivated in folate-free RPMI medium supplemented as described. H500 cells were grown in EMEM containing $1 \mathrm{mM}$ sodium pyruvate and $1 \mathrm{mM}$ nonessential amino acids in addition to FBS, glutamine, and antibiotics.

\section{Oligonucleotide and Oligonucleotide-Drug Combination Treatment In Vitro}

The phosphorothioate sequence specific antisense HER-2 oligodeoxyribonucleotides, (5'-TCC ATG GTG CTC ACT-3'), complementary to the initiation codon region of HER-2, and the control, nonsequence specific scrambled (5'-CTA GCC ATG CTT GTC-3') ODNs (SC ODN) were synthesized and purified by reverse phase HPLC by The Midland Certified Reagent Co. (Midland, TX, USA). The scrambled ODN has the same nucleotide composition as the antisense molecule, but in random order. Screening of both the antisense and scrambled ODN sequences against the GenBank Database indicated that the antisense ODN had homology only to HER-2; no homology was found between the scrambled ODN and any sequence in the database. The lyophilized ODNs were dissolved in sterile water to a concentration of $1 \mathrm{mM}$.

ODN-Lipofectin Reagent complexes were prepared as recommended by the manufacturer using Lipofectin Reagent (1 mg/ml; Life Technologies, Gaithersburg, MD, USA). Briefly, $7 \mu \mathrm{l}$ of Lipofectin Reagent was diluted into $100 \mu \mathrm{l}$ of serum- and antibiotics-free medium (IM) and allowed to stand at room temperature for $10 \mathrm{~min}$. The diluted Lipofectin Reagent was gently mixed with $100 \mu \mathrm{l}$ of a $30-\mu \mathrm{M}$ ODN solution, incubated for an additional $20 \mathrm{~min}$, and combined with $800 \mu \mathrm{l}$ of IM to yield $3 \mu \mathrm{M}$ of ODN. For transfection this solution was diluted with serum free medium to the desired ODN concentration.

The preparation of folate-cationic liposomes has previously been described (11). All of the lipids used were obtained from Avanti Polar Lipids (Alabaster, AL, USA). The folate-liposome-ODN complex was prepared as described for the Lipofectin Reagent, except that the molar ratio was $20 \mathrm{nmol}$ of folate-liposome to $2 \mathrm{nmol}$ of ODN in $1 \mathrm{ml}$ of folate-free medium.

MDA-MB-435 and MDA-MB-435 FF cells $(5 \times$ $10^{3}$ cells/well in $100 \mu \mathrm{l}$ of the appropriate medium) were plated in triplicates in a 96-well microtiter plate. Following overnight incubation, the cells were washed twice with serum-free medium, overlaid with $50 \mu \mathrm{l}$ of the transfection complex in serum-free 
medium and incubated for $12 \mathrm{hr}$, after which $50 \mu \mathrm{l}$ of the appropriate medium, supplemented with $20 \%$ FCS, $4 \mathrm{mM}$ L-glutamine, and antibiotics, were added. The cells were then incubated for an additional $12 \mathrm{hr}$, followed by the addition of $100 \mu \mathrm{l}$ of the appropriate supplemented medium, with or without drug, and incubation continued for approximately $72 \mathrm{hr}$. The chemotherapeutic drugs used were paclitaxel (Taxol, Bristol-Myers Squibb Co., Princeton, NJ, USA) and docetaxel (Taxotere, Aventis Pharmaceuticals Inc., Bridgewater, NJ, USA). After incubation for $72 \mathrm{hr}$ at $37^{\circ} \mathrm{C}$ in a humidified atmosphere containing $5 \% \quad \mathrm{CO}_{2}$, the wells were washed once with PBS and the cell viability XTTbased assay was performed according to the manufacturer's protocol (Boehringer Mannheim, Indianapolis, IN, USA). In the presence of an electron-coupling reagent, XTT (sodium 3' -[1-(phenylamino-carbonyl)3,4-tetrazolium]-bis(4-methoxy-6-nitro)benzene sulfonate), is converted into orange formazan by dehydrogenase in the mitochondria of living cells. The formazan absorbance that correlates to number of living cells was measured at $450 \mathrm{~nm}$ using a microplate reader (Molecular Devices, Menlo Park, CA, USA). The $I C_{50}$ was interpolated from the graph of the $\log$ of drug concentration versus the fraction of surviving cells.

\section{$5^{\prime}$ End $\left[^{35} S\right]$ or $\left[^{32} P\right]$ Labeling of ODNs}

$5^{\prime}$-end labeling of the ODNs was accomplished as described (15) using $\left[\gamma^{-35} \mathrm{~S}\right]$ ATP, $10 \mathrm{mCi} / \mathrm{ml}$ (Amersham, Piscataway, NJ, USA) or $\left[\gamma^{-32} \mathrm{P}\right]$ ATP, 6000 $\mathrm{Ci} / \mathrm{mmol}$ (NEN, Boston, MA, USA), and T4 polynucleotide kinase in a reaction buffer supplied with the enzyme (New England Biolabs, Beverly, MA, USA). Prior to phosphorylation, the ODN, in water, was heated at $95^{\circ} \mathrm{C}$ for $5 \mathrm{~min}$ and then immediately cooled on ice. After phosphorylation, the ODNs were purified by electrophoresis on a $19 \%$ polyacrylamide/urea gel and desalted using NAP-5 columns (Pharmacia Biotech, Piscataway, NJ, USA) as recommended (15).

\section{Uptake Studies}

In the context of our experiments, the term "uptake" refers to the amount of radioactivity associated with the cells after incubation with labeled ODN and after extensive washing with PBS. For determination of cellular uptake, the cells were seeded at $1 \times 10^{5}$ cells/well in a 6-well plate. $5^{\prime}\left[{ }^{32} \mathrm{P}\right]-$ labeled ODN was mixed with 5'-phosphorylated unlabeled ODN at a ratio of $1: 10(\mathrm{v} / \mathrm{v}$.$) . The cells$ were treated, 18-24 hr postseeding, with $2 \mu \mathrm{M}$ (equivalent to $10^{7} \mathrm{cpm} /$ well) of this ODN mixture either alone, or complexed with Lipofectin or folate-liposomes as described. After incubation, the medium was removed and the cells washed three times with excess PBS. The medium and PBS washes were pooled for scintillation counting to determine the amount of free ODN. The cells were then lysed with $0.5 \mathrm{ml} /$ well RIPA buffer ( $1 \%$ nonidet $\mathrm{P}-40,0.5 \%$ sodium deoxycholate, $0.1 \%$ sodium dodecyl sulfate [SDS], $0.1 \mathrm{mg} / \mathrm{ml}$ phenylmethylsulfonyl fluoride [PMSF], $30 \mathrm{mg} / \mathrm{ml}$ aprotinin, and $1 \mathrm{mM}$ sodium orthovanadate, all of which were obtained from Sigma Chemical Company, [St. Louis, MO, USA]). Cellular uptake was determined by Cherenkov counting of the washes and cellular lysate and was expressed as the percent of the total amount of the ODN added to the medium that was bound to $1 \times 10^{5}$ cells.

\section{In Vivo ODN Stability and Distribution}

MDA-MB-435 xenograft tumors were induced by subcutaneous injection of $2.5-3 \times 10^{6}$ cells into the mammary fat pad of female NCR nu/nu athymic nude mice. When the tumors reached approximately $100 \mathrm{~mm}^{3}$ the animals were intravenously injected once, via the tail vein, with 20 nmole $(\sim 4-5 \mathrm{mg} / \mathrm{kg})$ of either free AS HER-2 ODN or AS HER-2 ODN complexed with folate-liposome-2 (F-Lip-2). The F-Lip-2-AS HER-2-ODN complex was prepared for in vivo injection by mixing $300 \mathrm{nmol}$ of F-Lip-2 (in $150 \mu \mathrm{l}, 5 \%$ dextrose) with $20 \mathrm{nmol}$ of ODN (in $100 \mu \mathrm{l}$ water). After $20 \mathrm{~min}$ at room temperature, the volume was brought up to $300 \mu \mathrm{l}$, made to $5 \%$ dextrose, and injected into the tail vein.

To evaluate the stability of the ODN alone or in complex with F-Lip-2 in blood, 100-200 $\mu$ l of blood from the orbital sinus of each mouse was obtained prior to, and at various times from $30 \mathrm{~min}$ to $72 \mathrm{hr}$ postinjection, and placed in heparinized tubes. Two-thirds of each blood sample was centrifuged at $1000 \times \mathrm{g}$ for $7 \mathrm{~min}$. The plasma was removed and $100 \mu \mathrm{l}$ was incubated with $400 \mu \mathrm{l}$ of $50 \%$ acetonitrile (Sigma) for $30 \mathrm{~min}$ at room temperature. The mixture was centrifuged at $10,000 \times \mathrm{g}$ for $20 \mathrm{~min}$ at $4^{\circ} \mathrm{C}$. The supernatant was removed and the ODN precipitated by the addition of 10 volumes of cold $2 \% \mathrm{LiClO}_{4}$ (Sigma) in acetone, followed by centrifugation at $10,000 \times \mathrm{g}$ for $10 \mathrm{~min}$ at $4^{\circ} \mathrm{C}$. The resulting pellet was dissolved in $100 \mu \mathrm{l}$ of water, boiled for $3 \mathrm{~min}$ and immediately cooled on ice. Ten microliters of the ODN was $\left[{ }^{32} \mathrm{P}\right]$ postlabeled as described. The reaction mixtures were separated on a $19 \%$ polyacrylamide/urea gel and the bands visualized via autoradiography using Biomax MS film (Kodak, Rochester, NY, USA).

For the ODN distribution assay $\left[{ }^{35} \mathrm{~S}\right]$-labeled ODN (10 $\mu \mathrm{Ci}, 20 \mathrm{nmol})$, either alone or in complex with F-Lip-2 (total volume $300 \mu \mathrm{l}, 5 \%$ dextrose) prepared as described, were intravenously injected into the mice. Blood was taken from the orbital sinus and the tumors were excised at various times from $30 \mathrm{~min}$ to $72 \mathrm{hr}$ after injection. The tumors were flash frozen in liquid nitrogen and crushed while frozen. One hundred milligrams of crushed tissue or $100 \mathrm{ml}$ of plasma were incubated in $2 \%$ $\mathrm{NaOH}$ solution at $37^{\circ} \mathrm{C}$ for $16 \mathrm{hr}$ and subsequently neutralized by the addition of one-fifth the volume of $100 \mathrm{mM}$ ammonium bicarbonate. 
The amount of radioactivity in $10 \mu \mathrm{l}$ of each lysate was determined by liquid scintillation counting using Ultima GOLDTM XR cocktail (Packard Instruments, Meriden, CT, USA).

\section{In Vivo Tumor Growth Inhibition Studies}

MDA-MB-435 xenograft tumors of $70-100 \mathrm{~mm}^{3}$ were induced as described. ODN treatment consisted of intravenous tail vein injection of 20 nmole AS HER-2 or SC-HER-2 ODN ( 4-5 mg/kg) complexed to F-Lip-2, given either alone or in combination with docetaxel. The ODNs were administered as a bolus three times per week. Docetaxel (at $20 \mathrm{mg} / \mathrm{kg}$ in $100 \mu \mathrm{l}$ PBS) was also delivered intravenously three times per week, on the same day as the oligonucleotide. A total of 11 injections of both ODN and docetaxel were administered to the animals over the course of the experiment. The size of each tumor was measured using calipers by a third party in a blinded manner. All animal studies were performed in accordance with Georgetown University institutional guidelines for the care and use of animals.

\section{Results \\ Optimization of ODN Delivery into Cells}

Using Lipofectin, a commercially available liposomal reagent, to mediate transfection, we have previously shown that antisense HER-2 ODN can sensitize human breast cancer cells to the commonly used chemotherapeutic agent paclitaxel in vitro, resulting in increased cell death by apoptosis (16). More significantly, we found that this response to AS HER-2 ODN was independent of the level of HER-2 expression in the tumor cells (10). MDA-MB-435 cells have only a basal level of HER-2 expression (10), yet this cell line displayed a high degree of AS HER-2-induced chemosensitization. We have also shown that MDA-MB-435 cells treated ex vivo with AS HER-2 $(0.3 \mu \mathrm{M})$ and paclitaxel (1 $\mathrm{nM})$ do not develop tumors in nude mice (16). Thus, treatment with antisense HER-2 oligonucleotides has the potential to enhance the therapeutic effect of the paclitaxel in the clinic, whether or not HER-2 is overexpressed.

However, to be effectively used as a therapeutic agent a more efficient delivery system than Lipofectin-one that can be administered systemically to reach metastases as well as primary tumor-must be used. We have developed a cationic liposomal delivery system that uses folate as the targeting ligand. This complex has been successfully used for systemic delivery of wild-type p53 to various tumor cell types (11). The presence of a ligand such as folate markedly increased the transfection efficiency of the liposome complex resulting in increased sensitivity of human tumor cell xenografts to radiotherapy and long-term tumor elimination (11-13). We have found, via Western blot analysis, that the folate receptor levels in breast cancer cell lines (i.e., MDA-MB-435, MDAMB-231, MDA-MB-453, ZR-75-1, and SK-BR-3) are elevated compared to normal cell lines H500, HMEC, and MCF10 (data not shown). Therefore, we wished to determine if this targeted delivery system could also be employed to efficiently and preferentially deliver AS HER-2 oligonucleotides to breast tumor cells.

Optimization of the liposome composition for the tumor cell type is a critical factor in achieving high transfection efficiency. Therefore, five different folate liposome formulations, varying the composition as well as the ratios between the lipids, were tested for their ability to deliver AS HER-2 ODN to MDA-MB-435FF cells in vitro as determined by uptake of $\left[{ }^{32} \mathrm{P}\right]$-labeled ODN. This folate-independent cell line was used to avoid blocking the cellular uptake of the folate-liposome by the large amounts of folate in the medium. As shown in Figure 1, there were significant differences in uptake between the five liposome formulations, with F-Lip-2 (dimethyldioctadecylammonium bromide [DDAB]/L$\alpha$-dioleoylphosphatidyl ethanolamine [DOPE] at a 1/1molar ratio) resulting in the highest AS HER-2 uptake by the MDA-MB-435FF cells. After $25 \mathrm{hr}$ incubation, approximately $30 \%$ of the $\left[{ }^{32} \mathrm{P}\right]$-labeled AS HER-2 ODN delivered by F-Lip-2 was found to have been taken up by the cells. In contrast, the level of uptake facilitated by F-Lip-1 or F-Lip-3 was only approximately $10 \%$. AS HER-2 ODN uptake mediated by the F-Lip-2 complex was also compared to that mediated by Lipofectin and to the uptake of free ODN by MDA-MB-435FF cells. The results are shown in Figure 2. Whereas transfection with Lipofectin could increase ODN uptake as compared to free ODN, transfection using F-Lip-2 complexed to AS HER-2 ODN resulted in a dramatic increase in ODN uptake (approximately 5-fold)

Uptake of AS-HER-2 Oligonucleotides Complexed with Different Liposome Formulations

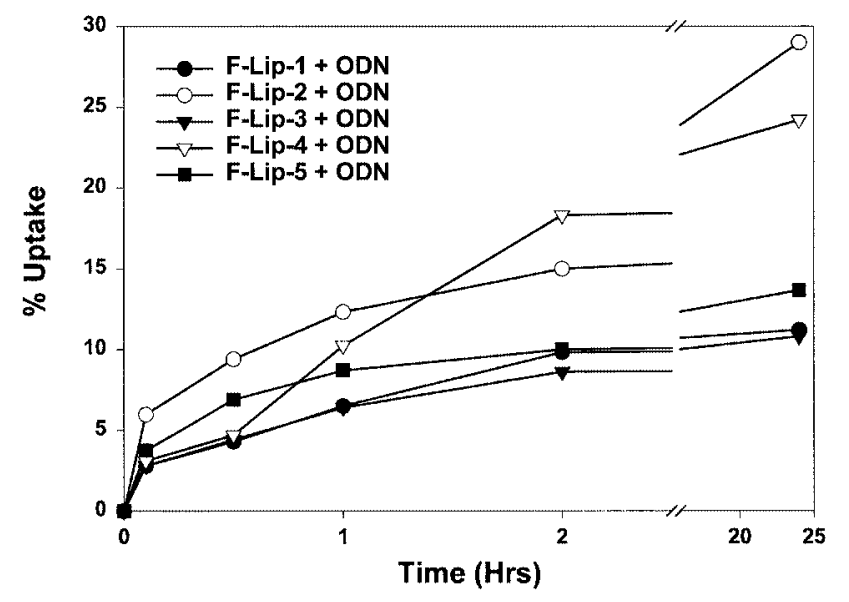

Fig. 1. Comparison of the uptake of AS HER-2 ODN complexed with five different liposome formulations in MDA-MB-435 FF cells. MDA-MB-435 FF cells, MDA-MB-435 cells grown in folate-free medium at least for 3 weeks. 
Comparison of AS-HER-2 Uptake Between LIPOFECTIN and F-Lip-2 In MDA-MB-435 Cells

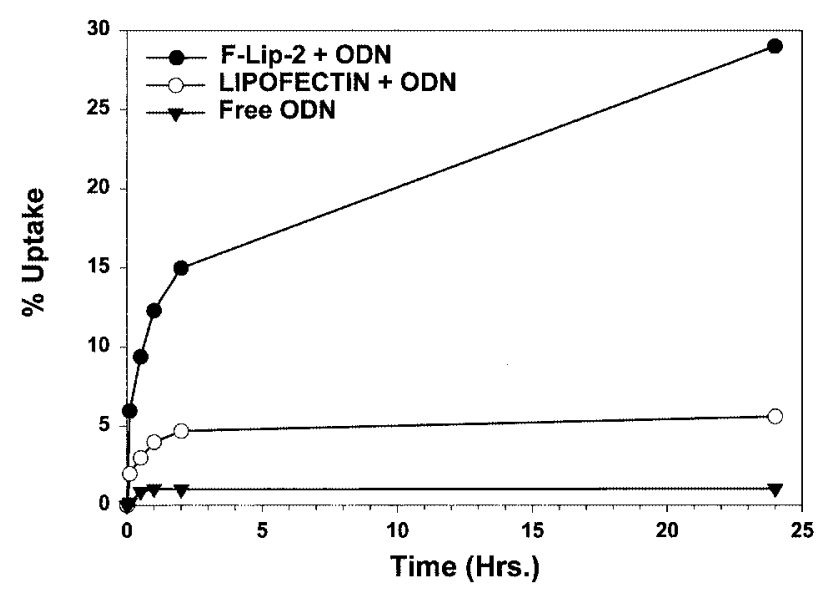

Fig. 2. Comparison of AS HER-2 ODN uptake mediated by F-Lip-2 versus Lipofectin or free ODN in MDA-MB-435 FF cells. Transfection with Lipofectin was performed as described in the manufacturer's protocol.

over that observed with the Lipofectin (less than $5 \%$ uptake). Therefore, analogous to our findings with plasmid DNA, the folate-liposome complex is capable, at least in vitro, of efficient delivery and transfection of AS oligonucleotides in breast tumor cells. Since our previous studies, our ligandliposome-plasmid DNA complex has shown good correlation between in vitro optimization and in vivo therapeutic efficacy (11); only the best complex, based on the above studies, was chosen for use in the remaining of the experiments described below.

\section{Drug Sensitization by AS HER-2 ODN}

Docetaxel, like paclitaxel, is a member of the taxane family. Docetaxel is one of the most active single chemotherapeutic agents for the treatment of metastatic breast cancer, and is now standard therapy in clinical practice $(17,18)$. Although paclitaxel and docetaxel have a similar mechanism of action (19), they are significantly different in clinical characteristics related to their efficacy/toxicity ratio relative to dose and schedule (18).

As mentioned, our previous in vitro antisense HER-2 chemosensitization studies (10) examined the effect of Lipofectin-delivered AS HER-2 ODN on the response of various breast cancer cell lines, including MDA-MB-435 to paclitaxel. Here we extended these studies to both taxane-based drugs. More significantly, we compared the level of in vitro sensitization of breast cancer cells achieved with AS HER-2 ODN delivered by either Lipofectin or our folate-liposome delivery system.

As with our earlier study, the AS HER-2 ODN employed in these studies is a phosphorothioate pentadecamer complementary to the initiation codon region of HER-2 mRNA. Previous studies using a series of AS HER-2 ODNs complementary to the area around and including the initiation codon indicated that this oligomer yielded the most efficient inhibition of HER-2 protein synthesis (data not shown). AS HER-2 ODNs targeted to other regions of the gene had previously been found to be less effective in inhibiting protein synthesis (E. Chang, unpublished data).

AS HER-2 concentrations of 0.1 and $0.3 \mu \mathrm{M}$ were tested. These two concentrations were chosen because they were both below the $I C_{50}$ value (the concentration of treatment agent resulting in a $50 \%$ decrease in viable cells) for AS HER-2 alone in MDAMB-435. This is important because high concentrations of AS HER-2 itself can also inhibit cell growth. We also observed previously that for MDA-MB-435 cells, AS HER-2 concentrations greater than $0.3 \mu \mathrm{M}$, when used in combination with a chemotherapeutic agent, resulted in such a high degree of cell death that an accurate determination of the $I C_{50}$ was not possible. Moreover, at concentrations above $0.3 \mu \mathrm{M}$ the scrambled control oligomer begins to demonstrate some nonspecific toxic effects. In the assays evaluating in vitro sensitization by F-Lip-2-AS-HER-2, MDA-MB-435FF cells, adapted for growth in folatefree medium, were used so that the high levels of folate in the medium would not inhibit the binding of the folate ligand in the complex to the cellular receptor to demonstrate proof of principle. However, it should be noted that all of the in vivo studies that will be discussed below use the same F-Lip-2-AS HER-2 ODN complex administered through the blood stream to mice bearing the parental MDA-MB435 xenograft tumors. It is important to note that the MDA-MB-435FF cells also have a low level of HER2 expression as was observed in the parental MDAMB-435 cells (data not shown).

The degree of sensitization to the drug is based on comparison of the $I C_{50}$ values after transfection with AS HER-2 ODN versus transfection with scrambled (SC)-HER-2 ODN (fold sensitization = $\left.I C_{50} \mathrm{SC} / I C_{50} \mathrm{AS}\right)$. The results are summarized in Table 1 and representative cell survival curves are

Table 1. Chemosensitization of MDA-MB-435/435 FF cells by AS HER-2

\begin{tabular}{|c|c|c|c|c|}
\hline \multirow{2}{*}{$\begin{array}{l}\text { AS HER-2 ODN } \\
\text { Concentration }\end{array}$} & \multicolumn{2}{|c|}{ Lipofectin } & \multicolumn{2}{|c|}{ F-Lip-2 } \\
\hline & $0.1 \mu \mathrm{M}$ & $0.3 \mu \mathrm{M}$ & $0.1 \mu \mathrm{M}$ & $0.3 \mu \mathrm{M}$ \\
\hline $\begin{array}{l}\text { Fold sensitization } \\
\text { to paclitaxel }\end{array}$ & 2.4 & 4.9 & 6.7 & 9 \\
\hline $\begin{array}{l}\text { Fold sensitization } \\
\text { to docetaxel }\end{array}$ & 2.2 & 17 & 7.2 & $>30$ \\
\hline
\end{tabular}

${ }^{*}$ Fold sensitization $=$ ratio of $I C_{50}$ for SC HER-2 ODN to $I C_{50}$ for AS HER-2 ODN. 
A Chemosensitization of MDA-MB-435 by LIPOFECTIN- AS-HER-2 ODN

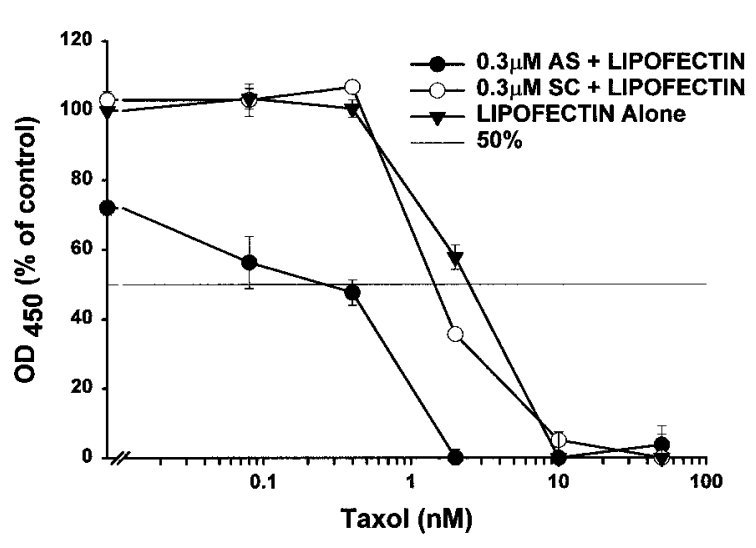

$\mathrm{IC}_{50}$ :

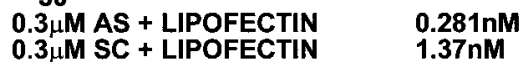
LIPOFECTIN Alone

Fold sensitization:

$\mathrm{IC}_{50} \mathrm{SC} / \mathrm{IC}_{50} \mathrm{AS}=4.9$

C Chemosensitization of MDA-MB-435 by LIPOFECTIN- AS-HER-2 ODN

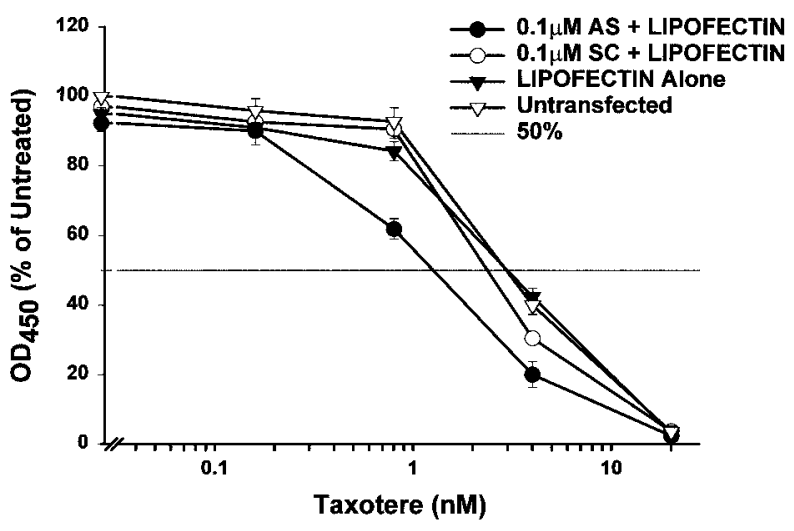

$\mathrm{IC}_{50}$ :

$0.1 \mu \mathrm{M}$ AS + LIPOFECTIN $\quad 1.2 \mathrm{nM}$ $0.1 \mu \mathrm{M}$ SC + LIPOFECTIN $\quad 2.6 \mathrm{nM}$ LIPOFECTIN Alone $\quad 3.1 \mathrm{nM}$ Untransfected
$3.1 \mathrm{nM}$
B Chemosensitization of MDA-MB-435 Cells by F-Lip-2- AS-HER-2 ODN

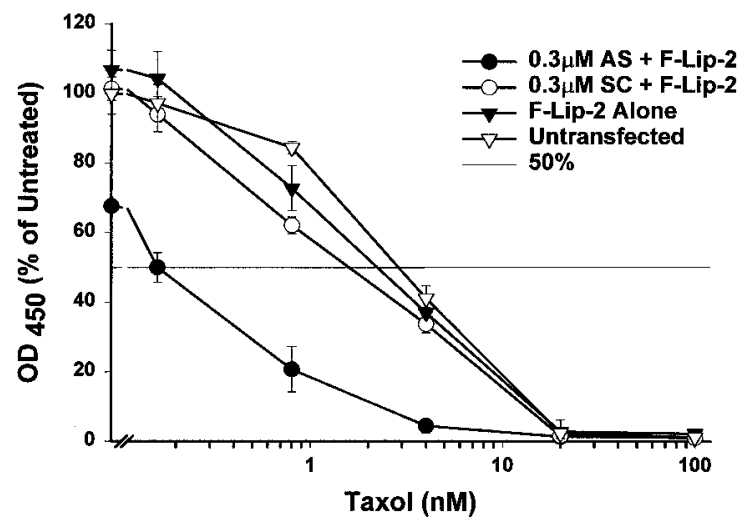

$\mathrm{IC}_{\mathbf{5 0}}$ :

$\begin{array}{ll}\text { 0.3 } \mu \mathrm{M} \text { AS + F-Lip-2 } & 0.15 \mathrm{nM} \\ 0.3 \mu \mathrm{M} \text { SC + F-Lip-2 } & 1.4 \mathrm{nM} \\ \text { F-Lip-2 Alone } & 2.1 \mathrm{nM} \\ \text { Untransfected } & 3 \mathrm{nM}\end{array}$

Fold Sensitization:

$\mathrm{IC}_{50} \mathrm{SC} / \mathrm{IC} \mathrm{C}_{50}$ AS $=9$

D Chemosensitization of MDA-MB-435 Cells by F-Lip-2-AS-HER-2 ODN

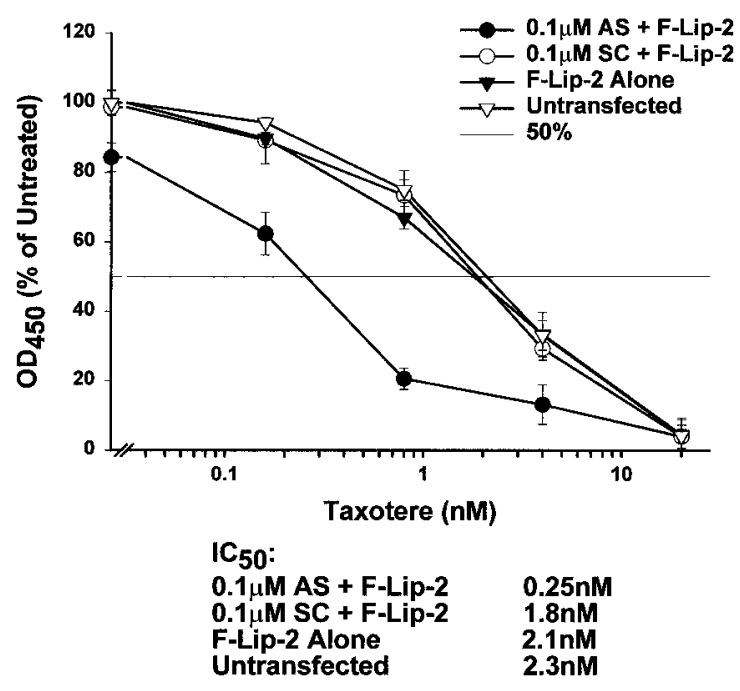

Fold Sensitization:

$\mathrm{IC}_{50} \mathrm{SC} / \mathrm{IC}_{50} \mathrm{AS}=7.2$

Fig. 3A-E. Comparison of the sensitization of MDA-MB-435/MDA-MB-435FF cells to paclitaxel (Taxol) and docetaxel (Taxotere) by FLip2-AS-HER-2 ODN or LIPOFECTIN ${ }^{\circledR}$-AS-HER-2 ODN. XTT assays were performed to assess the degree of sensitization to either Taxol or Taxotere after transfection with F-Lip2-AS-HER-2 ODN or LIPOFECTIN ${ }^{\circledR}$-AS-HER-2 ODN. Each point is the mean of triplicate samples \pm Standard Deviation. AS=Antisense-HER-2 ODN; SC=Scrambled HER-2 ODN; FOLD Sensitization=IC $\mathrm{IC}_{50} \mathrm{SC} / \mathrm{IC} \mathrm{C}_{50}$ AS. (A) Taxol sensitization with LIPOFECTIN ${ }^{\circledR}$ at an AS-HER-2 ODN concentration of $0.3 \mu \mathrm{M}$. (B) Taxol Sensitization with F-Lip-2 at an AS-HER-2 ODN concentration of $0.3 \mu \mathrm{M}$. (C) Taxotere sensitization with LIPOFECTIN ${ }^{\circledR}$ at an AS-HER-2 ODN concentration of $0.1 \mu \mathrm{M}$. (D) Taxotere sensitization by F-Lip-2 at an AS-HER-2 ODN concentration of $0.1 \mu \mathrm{M}$. (E) Taxotere sensitization by F-Lip-2 at an AS-HER-2 ODN concentration of $0.3 \mu \mathrm{M}$. 

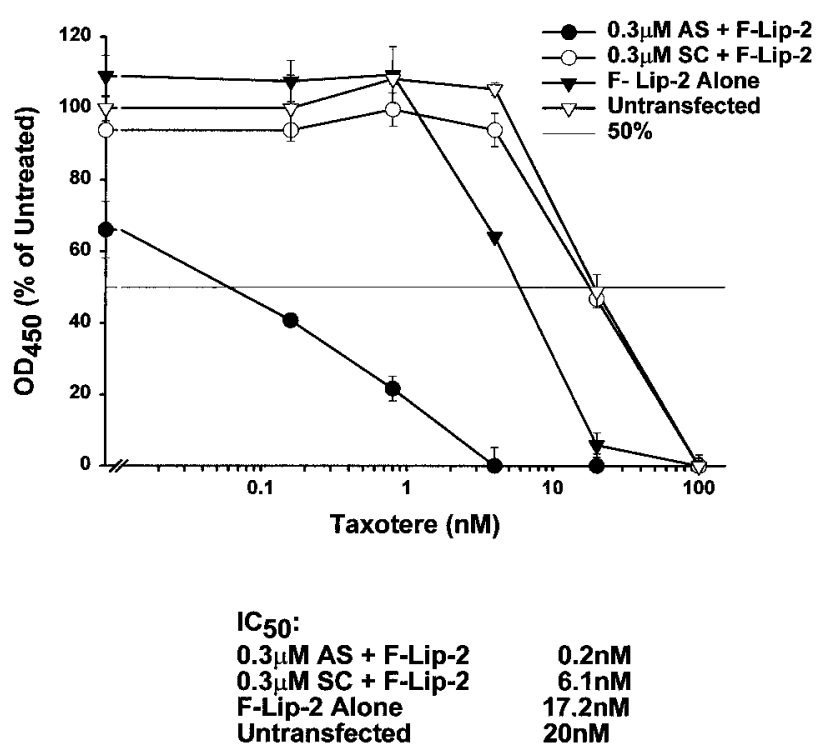

Fold sensitization:

$\mathrm{IC}_{50} \mathrm{SC} / \mathrm{IC} \mathrm{C}_{50} \mathrm{AS}=30.5$

Fig 3. (Continued)

given in Figure 3. As previously noted, only a minimal increase in sensitization is observed at an AS HER-2 concentration of $0.1 \mu \mathrm{M}$ using Lipofectin as the delivery vehicle (2.4- and 2.2-fold for paclitaxel and docetaxel, respectively). However, AS HER-2 ODN delivery mediated by the F-Lip-2 targeted delivery system resulted in a 6.7- and 7.2-fold increase in cell killing by paclitaxel and docetaxel, respectively, at the same low concentration of $0.1 \mu \mathrm{M}$. Although the level of sensitization via Lipofectin delivery was much higher at an AS HER-2 ODN concentration of $0.3 \mu \mathrm{M}$, particularly for docetaxel, the fold sensitization obtained using F-Lip-2 was approximately double the Lipofectin value. In fact, there was a 30 -fold increase in response of the cells to Docetaxel after treatment with the F-Lip-2-AS HER-2 complex (Fig. 3E). Thus, as demonstrated with plasmid DNA (11), this folatedirected liposomal delivery system can deliver antisense ODNs and efficiently transfect tumor cells in vitro resulting in increased effectiveness of the therapy.

Stability in Blood of AS HER-2 ODN in Complex with F-Lip-2

For a clinical cancer therapy to be most effective, it must be able to reach metastatic as well as primary disease. Therefore, being able to systemically (intravenously) deliver the therapeutic agent is essential. Free AS ODNs are known to have an extremely short $T_{1 / 2}$ in blood (0.5-1 hr) depending on the ODN dose $(20,21)$. This aspect has limited their potential use in cancer therapy. We demonstrated above the ability of the F-Lip-2 to efficiently deliver the AS HER-2 ODN in vitro resulting in increased sensitization of breast tumor cells to chemotherapeutic agents. In an earlier in vitro study using antisense ras ODN, we demonstrated that when incubated with cell culture, ODNs complexed with untargeted commercial Lipofectin are more stable than ODN alone (22). Our folate-liposome delivery system has been shown to be able to efficiently deliver plasmid DNA preferentially to tumors after systemic administration (11). Thus, it may be that this liposome delivery system can also protect the ODNs from being degraded and from nonspecific binding to protein while in circulation. Therefore, we next assessed the stability of the AS HER-2 ODN in complex with the F-Lip-2 in blood in vivo using a mouse model.

Equal amounts of free ODN or AS HER-2 ODN complexed with F-Lip-2 were injected into the tail vein of nude mice bearing MDA-MB-435 xenograft tumors. Blood samples were taken at various intervals, from $30 \mathrm{~min}$ to $24 \mathrm{hr}$. After isolating the ODN from the blood, as described in Methodology, it was postlabeled with $\left[\gamma^{32} \mathrm{P}\right]$ ATP and equal counts of the reaction mixture separated on a $19 \%$ polyacrylamide/urea gel. Comparing the intact ODN bands in the free ODN and the F-Lip-2 ODN samples, Figure 4 shows that within $30 \mathrm{~min}$, there is $80 \%$ less intact ODN present in the blood from the animal receiving the free ODN versus the F-Lip-2 complexed molecule, with only a faint band still present in the free ODN sample. In contrast, the intact AS HER-2 ODN band is still clearly evident at 1 and $3 \mathrm{hr}$ postinjection in the plasma from the animal receiving the FLip-2 complex. Moreover, a faint intact ODN band is

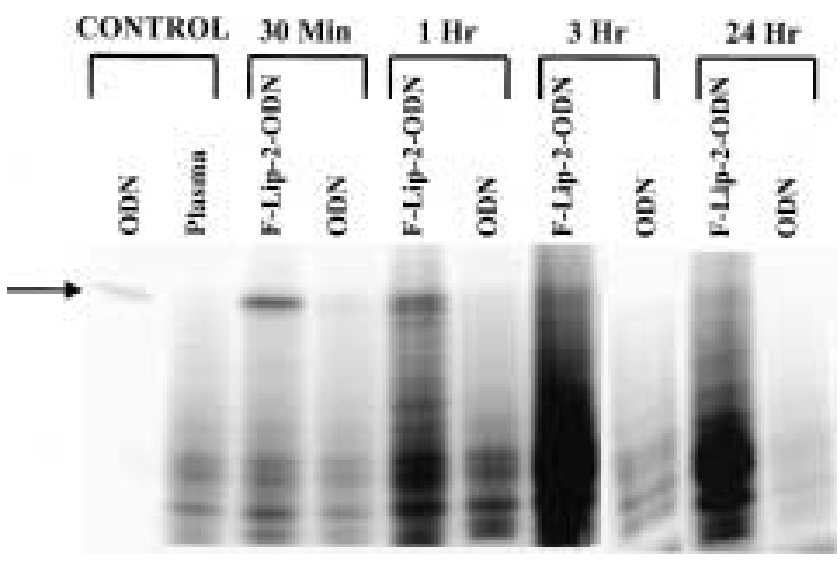

Fig. 4. Stability of AS HER-2 ODN in complex with F-Lip-2 in blood. Unlabeled ODN alone or ODN in complex with F-Lip-2 were intravenously administered once to mice. Blood was taken at various times from $30 \mathrm{~min}$ to $24 \mathrm{hr}$. The ODN from plasma was extracted, postlabeled with $\left[\gamma^{-32} \mathrm{P}\right]$ ATP and separated on $19 \%$ polyacrylamide/urea gel. Lane 1 (control ODN), pure $\left[{ }^{32} \mathrm{P}\right]$-labeled ODN run as a size standard. Lane 2 (plasma control), plasma from an untreated mouse. 
still visible even $24 \mathrm{hr}$ postinjection with the F-Lip2-AS HER-2ODN complex. Therefore, complexing the AS HER-2 ODN with our folate-liposome increases its stability in circulation. In addition, the persistence in circulation of the F-Lip 2-AS HER-2 ODN versus the free ODN was assessed using $\left[{ }^{35} \mathrm{~S}\right]-$ labeled AS HER-2 ODN either free or complexed to F-Lip-2 prior to a single intravenous tail vein injection. As determined by CPM, at each time point examined ranging from $0.5-72 \mathrm{hr}$, there was a 2- to 3-fold higher level of F-Lip-2-AS HER-2 ODN than free ODN evident in the plasma (data not shown). It should be noted that this liposome complex does not contain PEG and thus is not sterically stabilized. Therefore, a significant portion of the F-Lip 2-AS HER-2 ODN complex is cleared from the blood through binding to macrophages.

\section{Intratumoral Accumulation of $3^{35}$ SJ-Labeled ODN Complexed with F-Lip-2 After Systemic Administration}

We next evaluated whether the prolonged time in circulation evident with the F-Lip 2-AS-HER-2 complex resulted in increased uptake of the AS-HER-2 ODN in the tumor. $\left[{ }^{35} \mathrm{~S}\right]$-labeled AS-HER-2 ODN, alone or complexed to F-Lip-2, was injected intravenously once into the tail vein of nude mice bearing MDA-MB-435 xenograft tumors. The tumors were harvested $30 \mathrm{~min}$ to $72 \mathrm{hr}$ postinjection and treated as described in Methodology. The amount of $\left[{ }^{35} \mathrm{~S}\right]$ ODN present in $100 \mathrm{mg}$ of tumor tissue was compared between the free ODN and that complexed with F-Lip-2 over time. As evident in Figure 5, the AS HER-2 ODN complexed to F-Lip-2 has an increased level of accumulation in the tumor as compared to the free ODN at every time point examined. This was particularly true $6 \mathrm{hr}$ post-systemic delivery, where a greater than 6-fold higher level of the $\left[{ }^{35} \mathrm{~S}\right]$ ODN was present in

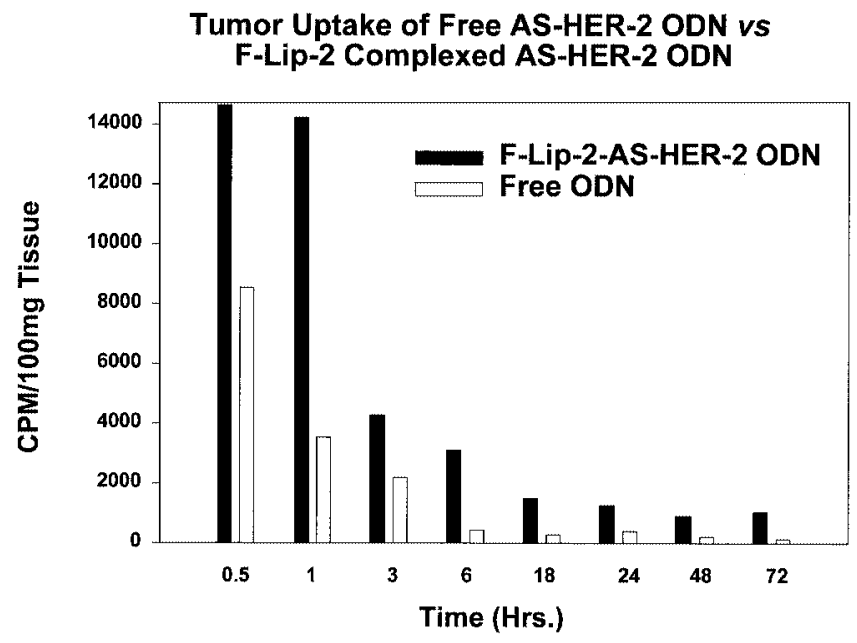

Fig. 5. Comparison of intratumoral accumulation of intravenously administered free $\left[{ }^{35} S\right]$-labeled ODN or $\left[{ }^{35} S\right]-$ labeled ODN complexed with F-Lip-2. Values given are CPM in $100 \mathrm{mg}$ of tumor tissue. Time is hours after intravenous injection. the tumor from the animal that received the F-Lip-2 complexed ODN compared to that injected with the free ODN. Moreover, a relatively stable level of the ODN was still present in the tumor 24,48 , and even $72 \mathrm{hr}$ postinjection of the F-Lip-2-AS Her-2 complex. Therefore, this prolonged uptake and presence in the tumor should permit the AS HER-2 to have a greater effect on tumor response to conventional cancer therapy.

\section{Effect of the Combination of Systemically Administered F-Lip-2-AS HER-2 ODN and Docetaxel on MDA-MB-435 Xenograft Tumors in an In Vivo Mouse Model}

The ability of the F-Lip-2-AS HER-2 to target and sensitize preexisting MDA-MB-435 xenograft tumors to the chemotherapeutic agent docetaxel in vivo was examined by assessing tumor growth inhibition (Fig. 6). Female athymic (NCR nu/nu) mice carrying MDAMB-435 mammary fat pad xenograft tumors of approximately $70 \mathrm{~mm}^{3}$ were intravenously injected, via the tail vein, with F-Lip-2-AS HER-2 (approximately $4-5 \mathrm{mg} / \mathrm{kg}$ of oligonucleotides) every other day to a total of 11 injections. A total of 11 intravenous doses of docetaxel (approximately $20 \mathrm{mg} / \mathrm{kg} /$ dose every other day) were also administered to the animals.

MDA-MB-435 Xenograft Tumors Treated with F-Lip-2-AS-HER-2 plus Taxotere (TXT)

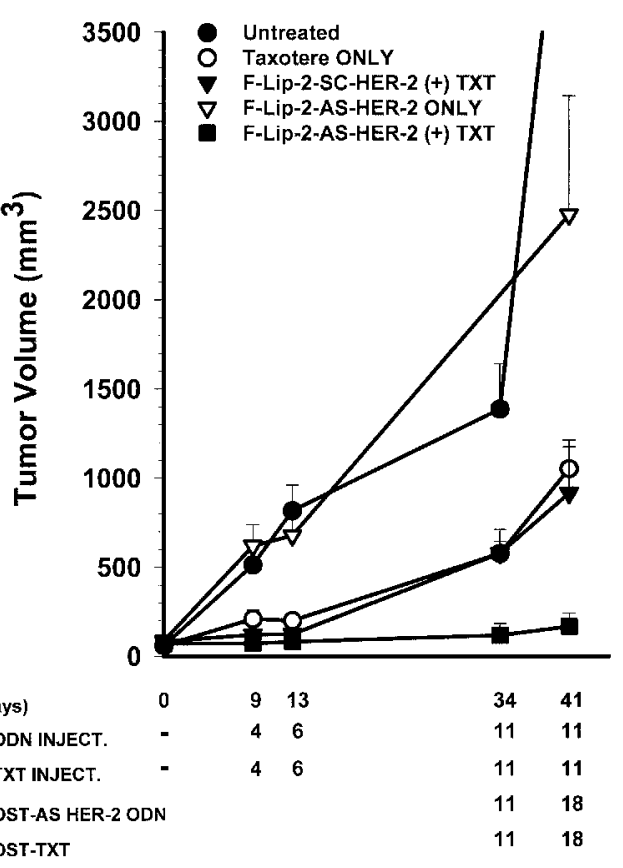

Fig. 6. Effect of the combination of F-Lip-2-AS HER-2 ODN and docetaxel on MDA-MB-435 xenograft tumors. Day 0 is the tumor volume $\left(\sim 100 \mathrm{~mm}^{3}\right)$ prior to initiation of treatment. F-Lip-2-AS HER-2 ODN and docetaxel were intravenously administered. Tumor volumes $\left(\mathrm{mm}^{3}\right)$ are the average of 4-10 tumors \pm SE. Error bars for the combination of F-Lip2-AS HER-2 ODN and docetaxel are too small to be visualized on the graph. 
Using dynamic light scattering (a Malvern Zetasizer 3000 ) the size of the F-Lip-2-AS HER-2 ODN was also measured. Based on the number value, the size of these particles is less than $100 \mathrm{~nm}$. This small size would facilitate their passage through the capillary bed into the tumor contributing to increased transfection efficiency. It is evident that this is a very aggressive breast cancer model. However, dramatic growth inhibition of the tumors was evident in the animals receiving the combination of F-Lip-2-AS HER-2. Moreover, although there was some docetaxel effect, these tumors began to rapidly increase in size after the cessation of treatment. Therefore, the systemically delivered, targeted liposome delivery of AS HER-2 oligonulceotides was clearly able to sensitize these tumors to the chemotherapeutic agent docetaxel, strongly inhibiting tumor growth even 3 weeks after the end of treatment.

\section{Discussion}

Because the first report that AS oligonucleotides could inhibit gene expression in a sequence-specific manner $(23,24)$, there has been tremendous interest in AS oligonucleotides as potential therapeutic agents. Their specificity make the use of AS molecules a rational strategy to selectively modulate the expressions of genes implicated in human diseases. Over the last 20 years, there has been a strong push by both pharmaceutical companies and researchers to develop these molecules into commercial therapeutics and AS oligonucleotides are now becoming significant components in gene therapy approaches, particularly cancer gene therapy. However, despite their early promise, only one antisense drug has reached the market. This drug, Vitravene ${ }^{\mathrm{TM}}$ (also called Fomivirsen), was approved in 1998 for the local (intravitreal) treatment of cytomegalovirus-induced retinitis in patients with AIDS (25). Although a number of other AS agents are in Phase I, II, and even Phase III clinical trials (reviewed in 26, 27), there are still significant hurdles to be overcome before they, as a group, can become first-line treatment regimens, including the problem of systemic administration. For this to be successful, the AS oligonucleotides must survive intact in sufficient quantity, and avoid protein binding long enough in the blood stream to achieve the desired level of gene inhibition or functional downregulation when they reach their target inside the cell $(26,28,29)$. In the majority of the current AS oligonucleotide clinical trials, the oligomers are being administered as free oligonucleotides. Free ODNs are rapidly degraded with a plasma half-life of only about $0.5-1 \mathrm{hr}$ (reviewed in 20,21 ). In vitro and in vivo studies have shown that as a consequence of this short half-life, the biological effects of free oligonucleotides are usually short lived, requiring repeated administration for sustained efficacy (reviewed in 26). For example, in Phase I clinical trials with ISIS3521, an AS phosphorothioate ODN against protein kinase
C alpha (PKC- $\alpha)$, the administration of the ODN ranged from a continuous 21-day intravenous infusion given every 28 days (30), to a $24-\mathrm{hr}$ continuous intravenous infusion once (31) and even a 2-hour intravenous administration three times per week for 3 out of every 4 weeks (32). Partial responses were evident in some patients in all of the studies. However, based on the published data for AS oligonucleotides in clinical trials with $b c l-2, r a f-1$, and $\mathrm{H}$-ras, in addition to PKC- $\alpha$, it appears that continuous infusion over 2-3 weeks is preferable to weekly administration with regard to both toxicity and effectiveness (33).

Due to the issues associated with administration of free oligonucleotides, carrier vehicles are being developed to improve systemic AS delivery. The most widely used method affording protection from nonspecific protein binding to the ODN while enhancing cellular delivery are liposomes, including cationic and $\mathrm{pH}$-sensitive fusogenic liposomes. Cationic liposomes are composed of positively charged lipid bilayers and can be complexed to negatively charged ODNs by simple mixing such that the resulting complex has a net positive charge. Features of cationic liposomes that make them versatile and attractive for DNA delivery include simplicity of preparation, the ability to complex large amounts of DNA, the ability to transfect many different cell types including nondividing cells, and low toxicity (34). From the perspective of human cancer therapy, cationic liposomes have been shown to be safe and effective for in vivo gene delivery with currently more than 75 approved clinical trials using cationic liposomes, 61 in the US alone $(35,36)$. At least six liposome based products are already on the market (37).

Because of their inherent positive charge, cationic liposomes have a high affinity for most negatively charged cell membranes. This makes them, so far, the most successful delivery method for ODNs (reviewed in 26, 38, 39). Perhaps the most significant factor to be considered regarding effective ODN delivery via cationic liposomes is optimization of the complex with respect to, among others, the type of lipids in the complex, their ratios, the ratio of lipid to nucleic acid, and even the method of formation of the complex. This is evident in the data presented here in Figure 1, where significant differences were noted in ODN uptake delivered by five different liposome complexes. There are numerous reports in the literature using cationic liposomes to deliver AS oligonucleotides in animal models. These include liposomes carrying AS oligonucleotides against the RNA component of human telomerase to treat prostate cancer xenografts (40) or gliomas in nude mice (41); an anti-TGF- $\alpha$ AS oligonucleotide to inhibit growth of squamous cell carcinoma of the head and neck xenograft tumors (42) and to prevent liver injury during endotoxemia (41); and anti-c-myc AS oligonucleotide to inhibit tumor growth and increase survival of mice bearing malignant melanoma xenograft tumors (42). AS-raf-1 ODN has also been 
successfully delivered intravenously and shown to radiosensitize human laryngeal carcinoma xenograft tumors in mice $(43,44)$ and is now in Phase I clinical trials (36). Phase I trials of intratumoral or intracavitary administered, liposome-encapsulated ElA in patients with breast/head and neck, or breast/ovarian cancers, respectively, that overexpress HER-2 have also been reported $(45,46)$. In addition, two other clinical trials using liposomes to deliver antisense molecules intratumorally against EGFR, or against IGF-1 for treatment of squamous cell carcinoma of the head and neck or glioblastoma, respectively, have been approved (35). However, in these two instances plasmids that code for an AS molecule rather than an ODN were encapsulated.

Although cationic liposomes can be used to systemically administer AS oligonucleotides, they are not inherently tumor specific. Therefore, in addition to increased accrual in the lung and liver, the potential exists for uptake by normal tissues with possible toxic side effects. In fact, some toxicity has been reported with oligonucleotide lipoplexes (28). It has been shown that the transfection efficiency of cationic liposomes can be dramatically improved by the addition of a ligand such as folate to the complex. Folate receptor levels are elevated in most cancer cells. We have seen elevated folate receptor levels in various breast cancer cell lines including MDA-MB435, MDA-MB-231, MDA-MB-453, ZR-75-1, and SK-BR-3 (M. S. Jhaveri, et al, submitted). We have previously shown that a folate-targeting lipoplex could preferentially and efficiently deliver its DNA package (plasmid DNA) to tumor cells resulting in a high degree of expression of the exogenous gene in the tumor cells, both primary and metastatic, with no histologic evidence of expression in normal organs such as lung, liver, or bone marrow (11). In addition, a liposome complex encapsulating an antisense ODN against the EGF receptor was targeted in vivo to, and induced growth inhibition of, $\mathrm{KB}$ cells by folate linked to the complex via PEG (47).

Therefore, to increase the tumor targeting ability of the systemically delivered AS HER-2-ODN lipoplex, we adapted our folate-lipoplex delivery system for use with AS ODNs. Targeting of the AS ODN lipoplex to tumors is an important consideration in developing efficient and effective AS ODN therapeutics. With tumor preferential uptake, less of the lipoplex should be necessary to demonstrate efficacy, possibly eliminating the need for the continuous intravenous infusions often required. The increased stability of the F-Lip-2-AS HER-2 ODN in circulation we observed will also potentially help in reducing the duration and frequency of treatment. This may improve the cost-effectiveness of this type of therapy. In addition, because the potential for uptake of the AS molecule by normal cells is significantly reduced using the targeted liposome-ODN complex, there is also the likelihood of reduced toxic effects on normal cells and tissues with a resultant decrease in adverse side effects for the patient. Uptake studies shown in Figure 5 demonstrate that there is increased uptake of the F-Lip-2-ODN complex in the tumor as compared to free ODN. Moreover, in the animal study presented here a dose of only 20 nmole ODN/injection, administered every other day for 3 weeks, was sufficient to effectively chemosensitize the MDA-MB-435 breast cancer xenografts to the drug docetaxel. This dose translates to only approximately $4-5 \mathrm{mg} / \mathrm{kg}$, an achievable dose in humans. The small size of the F-Lip2-AS HER-2 ODN complex $(<100 \mathrm{~nm})$ as measured by dynamic light scattering likely contributes to the effectiveness of this therapy. This small particle size would permit passage through the small capillaries and penetration further into the tumor and thus greater access to the tumor cells. In fact, using this folate-liposome system with a reporter gene we previously observed transgene expression 25 cell layers from the blood vessel in the tumor (11). It should be noted that although the in vitro experiments were performed in folate-free medium to demonstrate proof of principle, all of the animal stability, uptake, and efficacy studies were done under normal serum conditions in the mice. The results demonstrate that folate in the blood does not inhibit the targeting or transfection of the folate-liposome complex.

With regard to the observed chemosensitization, it is significant to note that the cell line used in these studies, both in vitro and to produce xenograft tumors (MDA-MB-435), does not overexpress HER-2. Despite this, the F-Lip-AS HER-2 complex was able to significantly sensitize these cells and tumors to paclitaxel and docetaxel both in vitro and in vivo. HER2 is believed to dimerize with the other three members of the ErbB family of growth factor receptors, ErbB-1, ErbB-3, and ErbB-4, being the preferential dimer-mate for each. These dimer complexes (which also include homodimers) form upon ligand binding, inducing their tyrosine kinase activity and triggering intracellular signaling through cell growth control pathways such as MAP-kinase $(1,48)$. Heterodimers containing HER-2 are apparently the most active. Klapper et al. (1) proposed that the ErbB family signaling network can be constitutively activated if one of the receptors is mutated or overexpressed. Thus, tumors that do not overexpress HER-2 may contain abnormalities in other family members resulting in aberrant cell growth signaling. Consequently, downmodulation of HER-2, the preferred heterodimer partner, could affect signaling through the ErbB family network in cases where it is the dimer partner (e.g., HER-3 or HER-4) rather than HER-2, which is abnormal. If so, then this opens the possibility of using anti-HER-2 therapies on more than just the $\sim 30 \%$ of human breast cancers that over express HER-2. This is supported by the data presented here with MDA-MB-435 xenograft tumors. Although this cell line does not overexpress HER-2 (10), it does overexpress HER-3 (C. Tang and M. Lippman, personal 
communication). These tumors were significantly sensitized by the F-Lip-2-AS ODN therapy to docetaxel, resulting in tumor growth inhibition even almost 3 weeks posttreatment.

We have shown that our tumor-targeted folate liposome complex can effectively deliver AS ODN systemically to tumors resulting in chemosensitization of breast cancer xenografts, irrespective of their HER-2 status. Moreover, we have previously shown that this ligand-liposome delivery system can target and affect metastatic as well as primary disease. Therefore, this delivery system can also be employed to treat metastatic breast cancer via AS HER2 ODN therapy. The ErbB family signaling network has such a significant role in controlling cell growth, it is likely to be a contributing factor in other types of cancer. In fact, abnormalities in HER-2 have been associated with ovarian, esophageal, and GI (including colon and pancreatic) malignancies. Thus, although breast cancer serves as a model, this tumor targeting and efficient downmodulation of HER-2 by our folate-liposome-AS HER-2 complex could also be applied to other tumor types, thus increasing the potential for AS ODN therapy to become a firstline clinical modality.

\section{Acknowledgments}

We thank Amy Marshall, Brianna Kalk, and Ekaterina Rait for their assistance in preparation of this manuscript; Ms. Wen-Hua Tang, and the Animal Research Shared Resources Facility for assistance with the animal studies. We also thank the Georgetown University Tissue Culture Core Facility and the Macromolecular Analysis Shared Resources Facility for their assistance. This work was supported in part by the National Foundation for Cancer Research Grant HU0001 (E. H. C.) and SynerGene Therapeutics, Inc. (K. F. P.).

\section{References}

1. Klapper LN, Kirschbaum MH, Sela M, Yarden Y. (2000) Biochemical and clinical implications of the ErbB/HER signaling network of growth factor receptors. Adv. Cancer Res. 77: 25-79.

2. Stancovski I, Sela M, Yarden Y. (1994) Molecular and clinical aspects of the Neu/ErbB-2 receptor tyrosine kinase. Cancer Treat. Res. 71: 161-191.

3. Slamon DJ, Godolphin W, Jones LA, et al. (1989) Studies of the HER-2/neu proto-oncogene in human breast and ovarian cancer. Science 244: 707-712.

4. Slamon DJ, Clark GM, Wong SG, Levin WJ, Ullrich A, McGuire WL. (1987) Human breast cancer: correlation of relapse and survival with amplification of the Her-2/neu oncogene. Science 235: 177-182.

5. Pegram MD, Finn RS, Arzoo K, Beryt M, Pietras RJ, Slamon DJ. (1997) The effect of HER-2/neu overexpression on chemotherapeutic drug sensitivity in human breast and ovarian cancer cells. Oncogene 15: 537-547.

6. Pegram M, Hsu S, Lewis G, et al. (1999) Inhibitory effects of combinations of HER-2/neu antibody and chemotherapeutic agents used for treatment of human breast cancers. Oncogene 18: 2241-2251.

7. Burris HA III. (2001) Docetaxel (Taxotere) plus trastuzumab (Herceptin) in breast cancer. Semin. Oncol. 28: 38-44.
8. Ring CJ, Blouin P, Martin LA, Hurst HC, Lemoine NR. (1997) Use of transcriptional regulatory elements of the MUCl and ERBB2 genes to drive tumour-selective expression of a prodrug activating enzyme. Gene Ther. 4: 1045-1052.

9. Ueno NT, Yu D, Hung MC. (2001) ElA: tumor suppressor or oncogene? Preclinical and clinical investigations of E1A gene therapy. Breast Cancer 8: 285-293.

10. Rait AS, Pirollo KF, Rait V, Krygier JE, Xiang L, Chang EH. (2001) Inhibitory effects of the combination of HER-2 antisense oligonucleotide and chemotherapeutic agents used for the treatment of human breast cancer. Cancer Gene Ther. 8: 728-739.

11. Xu L, Pirollo KF, Rait A, Murray A, Chang EH. (1999) Systemic $\mathbf{p} 53$ gene therapy in combination with radiation results in human tumor regression. Tumor Targeting 4: 92-104.

12. Xu L, Pirollo KF, Tang WH, Rait A, Chang EH. (1999) Transferrin-liposome-mediated systemic p53 gene therapy in combination with radiation results in regression of human head and neck cancer xenografts. Human Gene Ther. 10: 2941-2952.

13. Xu L, Pirollo KF, Chang EH. (1997) Transferrin-liposome-mediated p53 sensitization of squamous cell carcinoma of the head and neck to radiation in vitro. Human Gene Ther. 8: 467-475.

14. Xu L, Pirollo KF, Chang EH. (2001) Tumor-targeted p53 gene therapy enhances the efficacy of conventional chemo/radiotherapy. J. Control Release 6: 115-128.

15. Sambrook J, Fritsch EF, Maniatis T. (1989) Molecular Cloning: A Laboratory Manual, 2nd ed. Cold Spring Harbor Laboratory Press, Plainview, NY, USA.

16. Rait A, Krygier JE, Pirollo KF, Chang EH. (1999) Sensitization of breast cancer cells to taxol by antisense HER-2 oligonucleotides. Antisense Nucleic Acid Drug Dev. 9: 403-408.

17. Crown JP. (2001) The platinum agents: a role in breast cancer treatment? Semin. Oncol. 28: 28-37.

18. Nabholtz JM, Tonkin K, Smylie M, Au HJ, Lindsay MA, Mackey J. (2000) Chemotherapy of breast cancer: are the taxanes going to change the natural history of breast cancer? $E x$ pert Opin. Pharmacother. 1: 187-206.

19. O'Leary J VMWCMF. (1998) Taxanes in adjuvant and neoadjuvant therapies for breast cancer. Oncology 12: 23-27.

20. Agrawal S, Zhao Q. (1998) Mixed backbone oligonucleotides: improvement in oligonucleotide-induced toxicity in vivo. Antisense Nucleic Acid Drug Dev. 8: 135-139.

21. Glover J, Leeds JM, Mant T, et al. (1997) Phase I safety and pharmacokinetic profile of an intercellular adhesion molecule- 1 antisense oligodeoxynucleotide (ISIS 2302). J. Pharmacol. Exp. Ther. 282: 1173-1180.

22. Rait A, Uhlmann E, Peyman A, Will DW, Chang EH. (2000) Inhibition of Ras p21 synthesis by antisense undecamers with uniform and specifically arranged phosphorothioate linkages. Anticancer Drugs 11: 181-191.

23. Zamecnik PC, Stephenson ML. (1978) Inhibition of Rous sarcoma virus replication and cell transformation by a specific oligodeoxynucleotide. Proc. Natl. Acad. Sci. U.S.A. 75: 280-284.

24. Stephenson ML, Zamecnik PC. (1978) Inhibition of Rous sarcoma viral RNA translation by a specific oligodeoxyribonucleotide. Proc. Natl. Acad. Sci. U.S.A. 75: 285-288.

25. Crooke ST. (1998) Vitravene-another piece in the mosaic. $A n$ tisense Nucleic Acid Drug Dev. 8: vii-viii.

26. Akhtar S, Hughes MD, Khan A, et al. (2000) The delivery of antisense therapeutics. Adv. Drug Deliv. Rev. 44: 3-21.

27. Tamm I, Dorken B, Hartmann G. (2001) Antisense therapy in oncology: new hope for an old idea? Lancet 358: 489-497.

28. Akhtar S. (1998) Antisense technology: selection and delivery of optimally acting antisense oligonucleotides. J. Drug Target. 5: 225-234.

29. Juliano RL, Alahari S, Yoo H, Kole R, Cho M. (1999) Antisense pharmacodynamics: critical issues in the transport and delivery of antisense oligonucleotides. Pharm. Res. 16: 494-502.

30. Yuen AR, Halsey J, Fisher GA, et al. (1999) Phase I study of an antisense oligonucleotide to protein kinase $\mathrm{C}$-alpha (ISIS 
3521/CGP 64128A) in patients with cancer. Clin. Cancer Res. 5: 3357-3363.

31. Advani R, Fisher G, Lum B, et al. (2000) Coagulation and complement effects of an antisense phosphorothioate oligonucleotide targeting protein kinase C-alpha (ISIS 3521) are schedule and dose dependent. Proc. Am. Soc. Clin. Oncol. 17: 3586-3595.

32. Nemunaitis J, Holmlund JT, Kraynak M, et al. (1999) Phase I evaluation of ISIS 3521, an antisense oligodeoxynucleotide to protein kinase C-alpha, in patients with advanced cancer. $J$. Clin. Oncol. 17: 3586-3595.

33. Flaherty KT, Stevenson JP, O'Dwyer PJ. (2001) Antisense therapeutics: lessons from early clinical trials. Curr. Opin. Oncol. 13: 499-505.

34. Huang L, Viroonchatapan E. (1999) Introduction. In Non-viral Vectors for Gene Therapy. 3-22, Academic Press, San Diego, CA.

35. The Journal of Gene Medicine Clinical Trials Database. Available from: URL http://www.wiley.co.uk/wileychi/genmed/clinical. Accessed September 2001.

36. NCI Clinical Trials Web Site. Available from: URL http://www.clinicaltrials.gov.

37. Lian T, Ho RJ. (2001) Trends and developments in liposome drug delivery systems. J. Pharm. Sci. 90: 667-680.

38. Hughes J, Astriab A, Yoo H, et al. (2000) In vitro transport and delivery of antisense oligonucleotides. Meth. Enzymol. 313: $342-358$

39. Tari AM. (2000) Preparation and application of liposomeincorporated oligodeoxynucleotides. Meth. Enzymol. 313: 372-388.

40. Kondo Y, Koga S, Komata T, Kondo S. (2000) Treatment of prostate cancer in vitro and in vivo with 2-5A-anti-telomerase RNA component. Oncogene 19: 2205-2211.
41. Mukai S, Kondo Y, Koga S, Komata T, Barna BP, Kondo S. (2000) 2-5A antisense telomerase RNA therapy for intracranial malignant gliomas. Cancer Res. 60: 4461-4467.

42. Endo S, Zeng Q, Burke NA, et al. (2000) TGF-alpha antisense gene therapy inhibits head and neck squamous cell carcinoma growth in vivo. Gene Ther. 7: 1906-1914.

43. Gokhale PC, Soldatenkov V, Wang FH, et al. (1997) Antisense raf oligodeoxyribonucleotide is protected by liposomal encapsulation and inhibits Raf-1 protein expression in vitro and in vivo: implication for gene therapy of radioresistant cancer. Gene Ther. 4: 1289-1299.

44. Gokhale PC, McRae D, Monia BP, et al. (1999) Antisense raf oligodeoxyribonucleotide is a radiosensitizer in vivo. Antisense Nucleic Acid Drug Dev. 9: 191-201.

45. Yoo GH, Hung MC, Lopez-Berestein G, et al. (2001) Phase I trial of intratumoral liposome ElA gene therapy in patients with recurrent breast and head and neck cancer. Clin. Cancer Res. 7: 1237-1245.

46. Hortobagyi GN, Ueno NT, Xia W, et al. (2001) Cationic liposome-mediated ElA gene transfer to human breast and ovarian cancer cells and its biological effects: a Phase I clinical trial. Clin. Pharmacol. 19: 3422-3433.

47. Wang S, Lee RJ, Cauchon G, Gorenstein DG, Low PS. (1995) Delivery of antisense oligodeoxyribonucleotides against the human epidermal growth factor receptor into cultured KB cells with liposomes conjugated to folate via polyethylene glycol. Proc. Natl. Acad. Sci. U.S.A. 92: 3318-3322.

48. Tzahar E, Yarden Y. (1998) The ErbB-2/HER2 oncogenic receptor of adenocarcinomas: from orphanhood to multiple stromal ligands. [Review] [120 refs]. Biochim. Biophys. Acta 1377: M25-M37. 